\author{
Mariaelena Floriani
}

\title{
Natalia Ginzburg. \\ «Scrivere con il corpo» sul margine del «buco del Reale»
}

\begin{abstract}
Quest'articolo si propone di illustrare il legame tra corpo e scrittura che caratterizza la parabola creativa di Natalia Ginzburg (1916-1991) attraverso alcuni strumenti ermeneutici della psicanalisi lacaniana. Nella prima parte dell'articolo - Linguaggio e Inconscio - si definisce la natura del soggetto lacaniano, scisso tra parola soggettiva e struttura linguistica, tra il sé e l'illusione del sé: due poli divisi da un'opacità connaturata all'esistenza, che il linguaggio e la scrittura non possono risolvere. La seconda parte - La frammentazione come strumento narrativo e psicanalitico - indaga i modi in cui Natalia Ginzburg ha assunto su di sé la responsabilità di questa scissione trasformandola in contenente e contenuto di tutta la sua narrativa: da un lato, la frattura si manifesta tramite lo strumento della frammentazione, nelle diverse voci sperimentate dalla Ginzburg fra il 1942 e il 1984; dall'altro, la crepa diventa strumento psicanalitico-esistenziale per l'indagine del rapporto problematico tra soggetto e mondo. Ogni (non) presa di parola da parte dell'autrice o dei suoi personaggi è sintomo di un vuoto personalissimo e sempre diverso a seconda delle storie raccontate. Tenteremo di ricondurre la molteplicità di questi soggetti scissi all'unità tramite alcune delle pathosformeln descritte da Lacan, investendole di nuova luce. Nella terza parte - Scrivere con il corpo - si riassumono le strategie elaborate dalla scrittrice per rispondere al suo enjeux biografico, ovvero riuscire a scrivere malgrado l’infelicità causata dal rapporto con il «buco del Reale». Ogni parola vergata dalla penna di Natalia Ginzburg è una presa d'atto dell'intangibilità della vita e un atto di amore verso «l'opacità dell'enunciazione che soggiace agli enunciati che ne derivano».
\end{abstract}

The e-journal «altrelettere» is hosted at the URL: http://www.altrelettere.uzh.ch, in accordance with the Open Access Policy of the University of Zurich. Please cite this article as follows: M. FLORIANI, Natalia Ginzburg. «Scrivere con il corpo» sul margine del «buco del Reale», in «altrelettere», (2021), pp. 1-23, DOI: 10.5903/al_uzh-57.

(C) This article is licensed under a Creative Commons Attribution 2.5. Switzerland (CC BY-NC-ND 2.5). Please read the license terms on the website: http://creativecommons.org/licenses/by-nc-nd/2.5/ch/deed.en 


\section{Introduzione}

Nella prefazione a Cinque romanzi brevi e altri racconti, del 1964, Natalia Ginzburg celebra la chiusura di una stagione di "apprendistato" e traccia una retrospettiva della sua produzione, riconoscendo in Lessico famigliare il punto di arrivo della sua narrativa. Una frase di tale protostoria fungerà da fulcro della nostra riflessione, distinguendosi per la sua duplice potenza evocativa, à la fois esistenziale e tecnico-narrativa:

Non si trattava per me di diventare meno infelice ma di riuscire a scrivere malgrado la mia infelicità e senza curarmene, senza lasciare che intorbidisse le cose che scrivevo. Ma per riuscire è necessario che l'infelicità non sia in noi un'interrogazione lagrimosa e ansiosa bensì una consapevolezza assoluta, inesorabile, e mortale. ̀̀ necessario scrivere e pensare col cuore e con il corpo e non già con la testa ed il pensiero. (GINZBURG 2005, 15)

Il primo fatto da constatare è che la nostra (in)felicità o condizione terrestre ha una grande importanza per quello che scriviamo:

Quando noi siamo felici tendiamo a creare personaggi molto diversi da noi, a vederli nella gelida luce delle cose, estranee, [...] senza carità, con un giudizio scanzonato e crudele, ironico e superbo, mentre la fantasia e l'energia inventiva agiscono con forza in noi [...]. Quando siamo infelici abbiamo radici profonde e dolenti in ogni essere e in ogni cosa del mondo, del mondo fattosi pieno di echi e di sussulti e di ombre a cui ci lega una devota e appassionata pietà. (GINZBURG 2015b, 66-67).

In queste semplici righe la vocazione ginzburghiana prende parola e si fa corpo, dichiarando il suo amore per la vita. La scrittrice riesce a trascendere una sofferenza, un tormento di carattere autobiografico dopo aver accettato di vivere quel che c'è da vivere e di scrivere quel che c'è da scrivere, portando sulle spalle un peso atavico, indicibile e per questo universale: un macigno che non si sgretola sotto i colpi della logica e del raziocinio, che non si scioglie di fronte a quesiti dilanianti e sconfinati dolori. Quel macigno esiste ed essere uomini e donne libere significa scegliere di camminare nonostante quel peso, smettendo di pensare solo con il cervello e vivendo con il corpo nella concretezza del reale; abitandone il vuoto con l’imperativo di amarlo con il cuore. Natalia Ginzburg 
'scrive con il corpo' perché nei suoi romanzi la scrittura sfiora a livello epidermico le cose e le persone. Nessuno scavo psicologico incrina la limpidezza cristallina della parola. La scrittrice ha il garbo e l'intelligenza di non intellettualizzare le storie di vita perché le cose parlano da sé e quando tacciono il loro silenzio è più denso di qualsiasi discorso.

L'obiettivo di questo scritto consiste nell'approfondire il legame corposcrittura che caratterizza la narrativa ginzburghiana attraverso alcuni elementi cardine della psicoanalisi lacaniana e dello strutturalismo linguistico. I concetti teorici definiti nella prima parte del testo serviranno ad aprire un varco nella materia narrativa, al fine di rilevare le aperture saggistiche e gli espedienti tecnici che hanno ispirato la nostra lettura. Nella vitalità del processo testuale si trovano conglomerate, oltre a quella del lettore, diverse soggettività legate al piano esistenziale, sociale, politico e psichico in cui operava l'autrice. Isolare queste singole istanze permetterà di comprendere quale rapporto esse suggeriscano di instaurare con il 'buco del reale', nella consapevolezza che la loro peculiarità continua a vivere nella voce polifonica della Ginzburg.

\section{Linguaggio e inconscio}

Prima di addentrarci nel flusso di storie della narrativa ginzburghiana è necessario isolare alcuni concetti chiave della teoria psicanalitica di Jacques Lacan, la più complessa e problematica prosecuzione del lavoro di Freud sull'inconscio. La specificità e la novità delle sue formulazioni consistono nel dialogo serrato cui Lacan costringe la psicoanalisi freudiana e lo strutturalismo linguistico, rifacendosi al Cours de linguistique générale (1916) di Ferdinand de Saussure. La fusione e il parziale sovvertimento di queste due teorie porteranno al seguente assunto: l'inconscio è un effetto di linguaggio, privo di fissazioni di senso, e non più, come in Freud, cerniera tra il biologico e lo psichico. Lacan attiva la rottura dell'idea saussuriana del segno inteso come un tutt'uno di significante e di significato; non vi è più, insomma, rapporto di correlazione biunivoca tra i due elementi del segno, bensì una totale autonomia del significante che si identifica solo con la struttura linguistica e non con la parola soggettiva e individuale. Colui che scrive, parla o legge, è un soggetto diviso da una crepa che si è aperta tra il sé e l'illusione del sé, il cui limite è 
segnato da un vetro appannato. Risolvere questa opacità tramite il linguaggio tradizionalmente inteso è la più grande delle illusioni. La discrasia che nasce fra sguardo e visione, fra l'essere visti e il vedersi è la diretta conseguenza di un linguaggio internamente scisso che non può ricucire le fratture e ricondurre all'unità perduta: il soggetto si dovrà accontentare di una visione a brandelli, manchevole, insoddisfacente.

Così anche la scrittura, rappresentazione visiva, mediante segni grafici convenzionali di espressioni linguistiche, si trova intrappolata fra i due poli di un linguaggio scisso, quello che pertiene al significante e quello relativo al significato: il primo si situa dalla parte del Simbolico, il secondo dalla parte del Reale. Al registro del Simbolico fanno capo il linguaggio comune, ordinario e la sua struttura formale soggetta alle pressioni di un determinato potere linguistico, sociale, culturale, che qui chiameremo "ideologia"1. Al registro del Reale appartiene il dire individuale del soggetto, che Jacques Lacan definisce lalangue $^{2}$, legato al caos eterogeneo del corpo pulsionale. Esso consiste in una molteplicità indifferenziata di elementi che è impossibile ricondurre a un senso, «un godimento corporeo che non si risolve mai completamente nella simbolizzazione e nella sintassi» (BERGAMIN 2017, 92).

Questa distinzione permette forse di cogliere meglio quanto accennato sopra, ovvero che l'articolazione linguistica dell'inconscio e la struttura formale del discorso rappresentano un'imposizione: un lavoro di interpretazione infinito che si muove sulla catena articolata dei significanti, dove l'ultimo significante è sempre mancante. Su lalangue al contrario, nessuna decifrazione e nessuna elucubrazione sono possibili, giacché ciò che ne emerge sono soltanto lacerti, detriti senza senso, significanti fuori senso.

Questi pochi assunti della teoria lacaniana possono essere utili a illuminare i modi di scrittura della Ginzburg di una luce nuova. La distinzione fra Reale e Simbolico entra in risonanza con la tensione tra fisiologia e "ideologia" sottesa ai racconti giovanili del decennio 1930-1940 e al romanzo breve La strada che va in città (1942). Progressivamente questa tensione si risolve nell'importanza cruciale della solidarietà fra mente e corpo, di cui la scrittrice rende conto attraverso alcune figure chiave nel suo 'romanzo più romanzo', Tutti i nostri ieri (1952), e nel suo 'romanzo meno romanzesco', Lessico famigliare (1963). 
Il concetto di lalangue sarà invece sfruttato nell'ottica del dire e del non dire ginzburghiano, quell'alternanza di conversazione e sotto-conversazione che in Le voci della sera (1961) costituisce lo scheletro del romanzo. La lingua del corpo spiega le difficoltà di una critica che si trova di fronte alla narrativa di Natalia Ginzburg come davanti a una parete liscia e senza appigli; una superficie di depositi dove "amore e dolore non potrebbero essere espressi meglio che dal riserbo che li tace» (SCARPA 2014, 197). Il silenzio di fronte all'opacità del presente, l'impotenza della parola ad esprimerlo porteranno a una nuova stagione, quella dei romanzi romani o babilonesi ${ }^{3}$ del decennio 19701980, dove il linguaggio, così inadeguato, è tuttavia riconosciuto come unica opportunità dell'esistenza, ovvero imprescindibile mezzo per situarsi dentro un Reale infranto, decomposto in migliaia di schegge.

L'idea di uno slittamento continuo dei significanti e di una infinita triangolazione prospettica sul reale è alla base della frammentazione narrativa che la Ginzburg mette in atto nei romanzi Caro Michele (1973) e La città e la casa (1984). È quantomeno curioso che in quegli stessi anni figure di spicco dello strutturalismo quali Jacques Lacan e Roland Barthes abbiano contestato il trattamento cartesiano del linguaggio come oggetto monolitico, incurante dell'implicazione del soggetto nel suo oggetto di studio e di colui che produce il linguaggio, cioè del soggetto parlante. Contro l'opera letteraria come sostanza, il testo consiste in un processo al quale non è possibile trovare punti di arresto (BARTHES 1984, 59-60). Laddove l'opera subisce la sua stessa chiusura per mezzo dell'ermeneutica che mira alla ricostruzione dei significati segreti, il testo si svolge invece nel campo del significante, come rimando e rinvio infinito. Anche la lettura è un atto di esplosione e disseminazione di senso, dove il lettore tenta di sottrarsi al discorso comune, da cui pure dipende, e di operarvi una rottura, nella speranza di un approdo alla propria singolarità irriducibile. Negli ultimi romanzi di Natalia Ginzburg, la disseminazione diventa dissipazione di senso di fronte all'accadere dei fatti, insensati e freddi come eventi naturali. La coralità dei cosiddetti romanzi di mistica tribale ${ }^{4}$ si smembra - negli ultimi romanzi epistolari - in una fuga a più voci: lo specchio della rappresentazione è andato in frantumi. 


\section{La frammentazione come strumento narrativo e psicanalitico}

La frammentazione diventa per la Ginzburg l'espediente tecnico per raccontare, con un effetto di semplicità, la celata complessità della vita. Lo scrivere breve, rigoroso, antilirico dell'autrice è pura poesia, se crediamo con Italo Calvino che «la poesia sia far passare il mare in un imbuto; fissarsi uno strettissimo numero di mezzi espressivi e cercare di esprimere con quello qualcosa di estremamente intricato» (CALVINO 2015, 7).

La scrittrice dei racconti giovanili era animata da una fede profonda nei mezzi letterari tradizionali e compiaciuta della sua maestria nel caratterizzare i personaggi attraverso parole argute e ironiche oppure repertori di frasi ben congegnate. La sua speranza era di poter rispecchiare interamente nelle sue storie i destini umani elaborati e partoriti dalla sua mente creatrice; l'obiettivo era di far riflettere nella forma del racconto il senso profondo della vicenda narrata. Nel saggio Il mio mestiere la scrittrice ripercorre gli anni spesi a perfezionare la tecnica del racconto breve:

In quell'epoca ho visto una volta passare per strada un carretto con sopra uno specchio, un grande specchio dalla cornice dorata. Vi era riflesso il cielo verde della sera e io mi son fermata a guardarlo mentre passava con una grande felicità e il senso che avveniva qualcosa di importante [...]. A un tratto m'era sembrato che passasse l'immagine della mia felicità stessa, lo specchio verde e splendente nella sua cornice dorata. Per molto tempo ho pensato che l'avrei messo in qualche racconto, per molto tempo ricordare il carretto con sopra lo specchio mi dava voglia di scrivere. Ma non m'è mai riuscito di metterlo in nessun luogo e a un certo punto mi sono accorta che era morto in me (GINZBURG 2015b, 62).

Osservare il mondo esterno e descriverlo nel pensiero era divenuto un esercizio vacuo, poiché tutte le espressioni architettate e messe da parte erano «come un carico di cose imbalsamate, facce mute e parole di cenere, paesi e voci e gesti che non vibravano più» (ibid. 63) Nell'esilio a Pizzoli, dopo un sofferto silenzio che sancisce la rinuncia al mestiere di scrittrice per quello di madre, la Ginzburg ricomincia a scrivere come una che non aveva scritto mai: «le parole erano come lavate e fresche, tutto era di nuovo come intatto e pieno di sapore e di odore» (ibid. 65). 
Il lievito vitale di Pizzoli fa fermentare La strada che va in città, romanzo breve del 1942, in cui l'io del personaggio protagonista, Delia, si scinde per la prima volta individuando il proprio deuteragonista nella storia. Per meglio comprendere la portata di questo evento, si farà riferimento a quella che Jacques Lacan definisce la 'fase dello specchio'. Nello sviluppo infantile è in gioco una discrepanza, quando il bambino si guarda allo specchio, tra il suo corpo percepito come frammentario e l'immagine che lo specchio gli rimanda, che è al contrario un'immagine ideale, completa, non mancante. Nella fase dello specchio si costituisce l'Io, in cui il soggetto si aliena. Si tratta di una fase primordiale che prelude all'ingresso nel Simbolico e nel linguaggio formalmente costituito. L'Altro, che pertiene al registro del Simbolico, per Lacan mortifica il soggetto assoggettandolo al campo del significante:

Par l'effet de parole, le sujet se réalise toujours plus dans l'Autre, mais il ne poursuit déjà plus là qu'une moitié de lui-même. Il ne trouvera son désir que toujours plus divisé, pulvérisé dans la cernable métonymie de la parole (LACAN 1973, 183).

La pulsione è un concetto che lega il campo del significante con quello del corpo, dove però quest'ultimo individua una costruzione dell'immagine dell'io che deve al ruolo dell'Altro la sua composizione. Alla luce di quanto emerso si arricchisce la lettura viscerale a cui Cesare Garboli sottopone le protagoniste femminili ginzburghiane (GARBOLI 2012, 6). È indubitabile, infatti, che Natalia Ginzburg indaghi il rapporto fisiologico delle sue protagoniste con il mondo, ossia il loro tragitto di formazione, registrandolo in tappe concrete e materiali subitaneamente tradotte in corpo narrativo.

La prospettiva lacaniana arricchisce il livello epidermico del racconto, non per tradire la poetica della scrittrice, il cui più alto merito è quello di volare rasoterra sulle cose e le persone; ma per connotarla silenziosamente secondo degli schemi psichici incontrovertibili. A titolo d'esempio, potremmo riconoscere che, per Delia, l'Altro è il Nini e che il loro amore mancato è una mancata relazione con il Reale. Il maschio risulta essere un oggetto altro, non raggiunto e solo osservato, mentre il raggiungimento del (presunto) oggetto desiderato coincide con «l'impatto della Ragazza col mondo», uno strappo che fa nascere la vita dal sangue (GARBOLI 1993, 11). Parimenti Anna, la protagonista 
di Tutti i nostri ieri, capisce sulla propria pelle come la vita possa iniziare solo con la lacerazione, quando rimane incinta del suo vicino di casa:

Era sola, era sola e nessuno le diceva niente, era sola nella sua stanza col vestito macchiato d'erba e sgualcito e le mani che tremavano forte. Era sola col viso di Giuma che le dava uno strappo di dolore al cuore, e ogni giorno sarebbe tornata con Giuma fra i cespugli sul fiume, ogni giorno avrebbe rivisto quel viso coi ciuffi arruffati e le palpebre strette sugli occhi, quel viso che non aveva più né parole né pensieri per lei (GINZBURG 1996, 121).

Ł̀ importante notare come il momento dell'unione carnale dei due soggetti segni per Anna la chiusura del circuito comunicativo e la fine di ogni possibilità di comprensione. Al contrario, con il futuro marito Cenzo Rena è proprio il dialogo, e dunque il linguaggio, a oliare gli ingranaggi del rapporto:

Com'era difficile essere marito e moglie, non bastava dormire insieme e far l'amore e svegliarsi con la testa vicino, non bastava per essere marito e moglie. Essere marito e moglie voleva dire fare dei pensieri parole, di continuo fare dei pensieri parole e allora si poteva anche trovare non più strana una testa accanto a sé sul guanciale, quando c'era un libero fluire di parole che rinasceva fresco ogni mattina (ibid. 186).

Affinché l’incontro con l'altro sia foriero di gioia e benessere, i due registri del Simbolico e del Reale devono essere presenti in eguale misura. L'amore fra due soggetti risiede in un corpo pulsionale non domato dalla parola ma espresso parzialmente e instancabilmente tramite il dialogo con essa. Ginzburg non riduce la portata di questa verità all'universo femminile ma decide di applicarla anche a Cenzo Rena, nel momento in cui questi è vicino alla morte per tifo. Dipinto dall'autrice come coscienza morale del romanzo e salvatore di Anna, egli è spinto a cercare l’integrità della diade moglie/marito nella superficie di uno specchio intatto in cui le immagini dei due amanti si riflettono:

Le chiese se avesse ricordato ancora quel giorno che si erano guardati insieme nello specchio del barbiere, quel giorno avrebbero deciso di sposarsi e avevano dei brividi freddi ma si sentivano tutt'e due molto forti, aggressivi, liberi [...]. Ma quel giorno come sembrava lontano e chissà cos'era successo dello specchio di quel barbiere, chissà se c'era cascata sopra una bomba, se non moriva gli sarebbe piaciuto andare 
a vedere se quello specchio era ancora intero, specchiarcisi con lei un'altra volta (ibid. 264).

Abbiamo visto come nei due romanzi del 1942 e del 1952 Natalia Ginzburg cominci ad avvertire quella tensione del soggetto scisso che le impedisce di fare della sua letteratura uno specchio integro della vita. Dopo aver dato spazio all'Io esistenziale e narrativo in Lessico famigliare, nella speranza di poter maneggiare almeno i pezzi della sua vita nel modo più completo possibile, la scrittrice vi rinuncia e decide di elaborare dei mezzi tecnici ad hoc per incarnare nel corpo del testo la lacerazione insita nella relazione del soggetto con il mondo e del soggetto con sé stesso.

Lessico famigliare è un libro di memorie e un romanzo perché nasce dal ricordo delle parole, dal suono delle parole pronunciate nella storia della famiglia Levi: vi si scolpisce, insomma, il dire di tutti i suoi componenti. Ultima della famiglia, «Natalia sfrutta lo spazio vuoto in cui si ritrova per sviluppare un occhio e un orecchio infallibili nel captare ogni infimo dettaglio rivelatore della vita associata. Diventa il testimone secondario che tutto capisce restandosene acquattato in seconda fila» (FERRERO 2016). Consapevole di quanto sia difficile parlare di sé, la scrittrice opta per la prospettiva di un io defilato, ridimensionato fino alla sparizione.

Il paradosso è che a farsi carico di una rappresentazione famigliare vista con gli occhi di una bambina è una Narratrice dalla doppia funzione e dal doppio ruolo, da una parte spettatrice insignificante, dall'altra regista assoluta [...]. Essere adulti è una realtà percepita dalla Narratrice del Lessico, ultima di una famiglia numerosa, venuta a sedersi a tavolo quando i giochi erano già stati fatti, come un privilegio irraggiungibile [...]. Il mondo non ha peso per Natalia che ha la capacità di far vivere gli altri appiattendoli su una sola dimensione, inchiodandoli al proprio punto di vista e togliendoli alla loro totalità e integrità, togliendo loro l'illusione di essere come volevano e come credevano (GARBOLI 2014, 237-38).

Non più, dunque, l'io inventato che la scrittrice utilizzava nei suoi primi racconti brevi, fosse il testimone della disfatta famigliare di un amico come in Casa al mare del 1937 oppure una moglie avvizzita dalla finzione e stritolata dalla meschinità del senso comune come in Mio Marito, 1941. In questa fase, la 
conquista dell'io era conquista dell'altro da sé per sacro orrore dell'autobiografia. Soltanto con La strada che va in città la Ginzburg capisce che la memoria, matrice creativa e àncora di salvezza, non è casuale ma amorosa. E allora scrive di Delia con l'unica di pretesa di far scaturire l'invenzione dalla rimembranza. Questa ragazza dallo sguardo offuscato di bambina è incapace di decodificare la realtà e, alunna mediocre, impara a conoscerne i tranelli vivendola. Il personaggio di Delia funge da ponte narrativo per arrivare all'io demistificatorio a lungo cercato dalla scrittrice: vicino alla verità delle cose e non suo onnipotente possessore. Il passo successivo si compie dieci anni dopo nel romanzo corale Tutti i nostri ieri, dove anche la scrittura in terza persona è orchestrata in modo da apparire il meno obiettiva e assoluta possibile. Questa saga generazionale nasce grazie all'infiltrazione della Storia, con i suoi drammi e le sue tragedie, nelle storie dei personaggi, che ne hanno una percezione parziale, intrecciata alle emozioni di quel preciso momento del loro vissuto. La voce narrante si fonde con la materia narrativa fino a diventare indistinguibile dai punti di vista degli attori: la prospettiva soggettiva dell'io permane, seppur velata da quella terza persona che presto diventerà impossibile.

La scrittura del soggetto diviso è portata a compimento con le sperimentazioni teatrali e i romanzi epistolari degli anni '70:

Ho scelto la forma epistolare perché volevo un io che corrispondesse a un lui; una prima persona con tante sfaccettature, molteplice. Le lettere sono questo. Invece di un solo io che racconta gli altri e se stesso, molti io che si raccontano (GINZBURG 2019, 247).

Caro Michele, romanzo semi-epistolare, segna la fine dell'autobiografismo della scrittrice risolvendo l'annoso dramma dell'io e dell'egli. Si tratta di una mediazione tra coralità e versione estremizzata di un narratore impersonale, punteggiata da inserti narrativi: da un lato, la scrittrice affida tali inserti solo al dialogo, dove la voce narrante scompare; dall'altro, uno sguardo puramente denotativo si limita a registrare ciò che sta alla superficie della realtà. Il baricentro del racconto è vuoto e sommariamente riempito dalle proiezioni elaborate dai personaggi su questa assenza. Le lettere sono accostate come 
documenti che «s'illuminano e si contraddicono a vicenda senza che la voce narrante debba incaricarsi di farlo» (SCARPA 2019, 259). Scritto dopo dieci anni di silenzio narrativo, il testo parla di una crisi dei modi di rappresentazione della realtà; misura dell'impotenza della parola e della solitudine che ne deriva.

La città e la casa segna l'apice più drammatico e nello stesso tempo l'accettazione dei limiti del linguaggio rispetto alla complessità del Reale. Il risvolto di sovraccoperta del romanzo ci illumina a questo proposito:

Mi auguro e spero che, nelle vicende e nelle fisionomie delle persone che si scambiano queste lettere, possa riflettersi un poco della vita dei nostri giorni. Ma la vita dei nostri giorni trovo che sia difficile da raccontare. Perciò se vi si rifletterà, vi si rifletterà in modo esiguo, estremamente frammentario e parziale, e come nelle schegge di uno specchio rotto. Devo dire che scrivendo romanzi, ho sempre avuto la sensazione d'avere in mano degli specchi rotti e tuttavia sempre speravo di poter ricomporre finalmente uno specchio intero. Ma non mi è mai successo e via via che andavo avanti a scrivere la speranza si allontanava. Questa volta però, fin dal principio, non speravo nulla. Lo specchio era rotto e io sapevo che ricomporne $\mathrm{i}$ pezzi mi sarebbe stato impossibile. Non avrei mai avuto il bene di avere davanti a me uno specchio intero (GINZBURG 2019, 269).

La concezione stendhaliana del romanzo come specchio condotto lungo la via cade nel momento stesso in cui viene riconosciuto il dramma del soggetto diviso tra visione e sguardo. Natalia Ginzburg lo riconosce per sé, per i suoi personaggi ma anche per i suoi lettori. Il soggetto, infatti, non è più il punto geometrico a partire dal quale viene colta la prospettiva del quadro. Il quadro si dipinge nell'occhio dello spettatore ma, allo stesso tempo, è lo spettatore a trovarsi inscritto nel quadro.

Ognuno dei personaggi esprime per lettera un giudizio sferzante, un'opinione soggettiva sugli individui che compongono la tribù, condizionando in maniera apparentemente decisiva l'opinione del lettore, persuaso della verità di quelle parole; ma le lettere si susseguono, le storie si intrecciano, le opinioni si confondono e si smentiscono l'una l'altra. Da questa disseminazione di senso deriva l'incertezza di fronte all'indicibilità del reale, sempre altro rispetto al dire individuale che cerca di imbrigliarlo. Il soggetto diventa allora parassita per l'impossibilità di staccarsi dalla sua visione personalissima dello stato delle cose; per la sua totale incapacità ad avere una presa su di esse. È dunque l'io 
grammaticale, da cui origina la scrittura, che nel suo manifestarsi in linguaggio diventa parassita di sé stesso. L'utilizzo del concetto di parassita nella teoria di Jacques Lacan è spiegato da Jacques-Alain Miller come segue:

Évidemment les élucubrations sont parasites, la vérité parasite, la parole elle-même est parasite; c'est une position sage, d'une sagesse qui prend en contre-pied la sagesse commune, la sagesse commune, qui fait fond sur le langage pour tamiser tout ça. La sagesse qui consiste à vous apprendre que vous pouvez vivre en bonne entente, en bonne intelligence, en harmonie avec la jouissance. C'est une sagesse subversive qui nous explique qu'il y a un parasite qui ne se laisse pas éliminer et qu'on peut seulement modifier, transformer, car ce qui est homme fait grouiller les parasites (BERGAMIN 2017, 250).

Dopo il 1963, l'io narrante diventa parassita: Natalia Ginzburg scappa da quell'io odioso e da quell'egli impossibile che le impedivano di salire sulla cima della montagna e di guardare dall'alto. Si potrebbe dire in altre parole che nel decennio 1960-1970 la scrittrice matura la consapevolezza di essere quel paesaggio che contempla, un paesaggio che esce a strappi da una visione continuamente interrotta: come quando si attraversa in auto un lungo viadotto i cui piloni oscurano a istanti alterni il godimento procurato dalla contemplazione della natura circostante. Il corpo si configura allora come contenitore e contenuto, come punto da cui la visione origina ma anche come goccia contenuta nell'insieme, talmente liquido e inafferrabile da apparire indicibile.

Tutto quello che la Ginzburg è stata fino allora non le interessa più [...]. Lo scrittore si eclissa, si mette da parte, in un angolo, e ascolta il coro di tante voci disperse, il fraseggio lasciato nell'aria da tutta la vita che non è stata sua. Non c'è bisogno di farsi domande. Il loro grido non si sente più, si è perso come un volo di uccelli mentre la vita correva e diventava adulta. In gioco non è più l'Altro, ma il Sé, la musica del Sé (GARBOLI 1993, 12).

Questo parassita insito nel dire microscopico della scrittrice si riverbera macroscopicamente sulla concezione del proprio mestiere: 
[...] questo mestiere non è mai una consolazione o uno svago. Non è una compagnia. Questo mestiere è un padrone, un padrone capace di frustarci a sangue, un padrone che grida e condanna. Noi dobbiamo inghiottire saliva e lacrime e stringere i denti e asciugare il sangue delle nostre ferite e servirlo. Servirlo quando lui ce lo chiede. Allora anche ci aiuta a stare in piedi, a tenere i piedi ben fermi sulla terra, ci aiuta a vincere la follia e il delirio [...]. I giorni e i casi della nostra vita, i giorni e i casi della vita degli altri a cui assistiamo, letture e immagini e pensieri e discorsi, lo saziano e cresce in noi. È un mestiere che si nutre anche di cose orribili, mangia il meglio e il peggio della nostra vita, i nostri sentimenti cattivi come i sentimenti buoni fluiscono nel suo sangue. Si nutre e cresce in noi (GINZBURG 2015b, 68-69).

Insomma, un parassita nel senso lacaniano del termine, un microbo che accompagna la Ginzburg per tutta la vita senza darle nessuna consolazione, nessuna tregua, permettendole tuttavia di sorreggere il suo corpo lungo il cammino, con $i$ piedi ben fermi sulla terra. È un mestiere che si radica nella fisicità dell'esistenza imponendo al corpo di scriverla e, in questo modo soltanto, di salvarla.

\section{Scrivere con il corpo}

Abbiamo constatato come la questione del soggetto diviso sia profondamente connessa a quella del dialogo e della parola. Le voci della sera, romanzoconversazione del 1961, è lo scritto che più si avvicina al problema della comunicazione tra esseri umani legati da vincoli sociali o affettivi:

È una storia di persone che cercano di sotterrare i pensieri, d'identificarsi soltanto nei gesti che compiono e nelle parole che dicono e finiscono per ritrovarsi strette in una morsa di assurdità e dolore. [...] Quel mondo sicuro della famiglia che i narratori dell'Ottocento rappresentavano come una realtà naturale è ormai qualcosa che può essere rievocato solo da una nostalgia mista a ferocia. Lo scrittore che è detto naïf è in verità affatto ingenuo o floreale: è prima di tutto caparbio e feroce e sbigottito e disperato. La letteratura comincia là dove si è andati molto più in là con l'odio o con l'amore per il genere umano. Comincia quando la volgarità quotidiana non ci raggiunge più e sentiamo solo la musica che scorre in fondo, sotto tutte le parole e tutti i gesti (CALVINO 2015, 5-9). 
Uno dei modi più pregnanti della scrittrice per rendere esplicito questo rapporto chiaro, asciutto, di condanna non invischiante, di familiarità non colpevole col mondo è l'utilizzo del dialogo; il far succedere tutto tramite le parole che i personaggi dicono. Le voci della sera è una architettura sonora $\mathrm{e}$ vocale di diversi racconti che abitano l'uno accanto all'altro e l'uno dentro l'altro, registrati ed emessi dalla voce di Elsa, sorta di camera di risonanza delle voci degli altri. L'unica storia in cui la narratrice-testimone è interamente implicata è la relazione con Tommasino, che Elsa evoca tramite una serie di recuperi memoriali individuali inframezzati da recuperi memoriali collettivi, di paese. La storia di questi due innamorati viene riassorbita senza far rumore in uno schema circolare e sempre uguale a sé stesso, che la coralità testimonia.

Tra i due giovani la comunicazione si articola secondo due parametri: quello della conversazione e quello della sotto-conversazione. La prima è quotidiana, funzionale al qui e ora e si serve del dialogo; la seconda riguarda tutte le cose sepolte nel non detto, nel silenzio. Lo scarto fra i due parametri comunicativi si rende visibile da sé: la Ginzburg giunge al gelo della tragedia attraverso il calore del corpo, ossia il linguaggio.

I nostri personaggi parlano così. Parlano così per ingannare il silenzio. Parlano così perché non sanno più come parlare [...]. Strappate dolorosamente al silenzio, vengono fuori le poche, sterili parole della nostra epoca, come segnali di naufraghi, fuochi accesi tra colline lontanissime. Flebili e disperati richiami che inghiotte lo spazio (GINZBURG 2015b, 71).

Durante gli incontri clandestini a Torino, Elsa e Tommasino stentano a capirsi: i loro dialoghi camminano sul posto. Lui vive in una stasi esistenziale, dimostrando scarsa aderenza alla vita. Un'eterna adolescenza, accompagnata dall'incapacità di mediare con la realtà, lo porta a fare affidamento sulla grazia di una relazione continuamente rinnovabile e revocabile. La presa di posizione femminile e la richiesta di stabilità, derivate dall'autentico sentimento che Elsa prova per Tommasino, porteranno alla crisi:

Ho sentito che non potevo più scegliere, che dovevo venire da te senza scampo [...] e fare tutto quello che tutti si aspettavano che io facessi, quello che anche tu con gli 
altri ti aspettavi da me. Così, ho preso a sotterrare i miei pensieri. Non potevo più guardare in faccia la mia anima. Per non sentire gridare la mia anima, ho girato le spalle, ho camminato lontano da lei". La relazione che avevamo "era qualcosa di molto leggero, di molto fragile, pronto a disfarsi al primo soffio di vento. Era qualcosa che non si poteva acchiappare, portare alla luce, senza che morisse. L'abbiamo portato alla luce, ed è morto, non lo riavremo mai più". (GINZBURG 2015a, 93).

Nel momento della vera apertura del circuito comunicativo, i due amanti si trovano di fronte alle verità di ciascuno, ognuna incompatibile con quella dell'altro. L'incontro risulta impossibile e nessuna convenzione sociale, nessun accordo a posteriori può camuffarne il mancato avvenimento. Per quale ragione l’incontro non è avvenuto? Perché quello che Tommasino nutriva per Elsa non era vero amore.

Secondo Jacques Lacan, amare è dare ciò che non si ha a chi non lo vuole. Se si ama una persona si desidera donarle ciò che ci manca e parimenti lei vorrà per noi il Bene, cioè quello che lei stessa considera fondamentale per essere felice. Ma la persona che ti ama non vuole per sé quello che tu vuoi per te. Vuole per te quello che le manca. Essere consapevoli di questo incrocio di impossibilità e frustrazioni è il primo passo verso l'accettazione dell'altro e la possibilità di amarlo per quello che è, con le sue verità e le sue idiosincrasie.

A questo punto, una domanda sorge spontanea. Se l'essere umano si trova continuamente scisso per l'impotenza della parola e per il buco del Reale che si trova nel corpo, come può riuscire nel mestiere di vivere? Natalia Ginzburg prova la strada della psicanalisi rimanendone, in definitiva, delusa.

Quando andiamo a farci psicanalizzare, ci dicono che dobbiamo smetterla di odiare così fortemente la nostra persona. Ma per liberarci di questo odio, per liberarci del senso di colpa, del senso di panico, del silenzio, ci viene suggerito di vivere secondo natura, di abbandonarci al nostro istinto, di fare della nostra vita una pura scelta. Ma fare della vita una pura scelta non è vivere secondo natura: è vivere contro natura, perché all'uomo non è dato scegliere sempre: l'uomo non ha scelto l'ora della sua nascita, né il proprio viso, né i propri genitori, né la propria infanzia: l'uomo non sceglie, di solito, l'ora della sua morte. L'uomo non può che accettare il suo proprio destino: e la sola scelta che gli è consentita è la scelta fra il bene e il male, fra il giusto e l’ingiusto, fra la verità e la menzogna (GINZBURG 2015b, 74). 
La strada che la Ginzburg propone di percorrere, visto che non ci è dato scegliere se essere felici o infelici ma ci è dato «scegliere di non essere diabolicamente infelici» (ibid. 75), è quella di ‘scrivere con il corpo'. In primo luogo, dire che il corpo genera la scrittura significa evidenziare che c’è qualcosa, un soggetto in quanto corpo, che si oppone al linguaggio. Si deve far fronte al contempo all'estraneità del linguaggio rispetto al soggetto e, viceversa, all'estraneità del soggetto rispetto al linguaggio.

In questo senso, la poetica della Ginzburg mette in evidenza la condizione di un soggetto non solo mortificato dal linguaggio che lo governa dall'esterno, ma anche abitato da un linguaggio altro, più corporeo, che dall’interno vanifica ogni suo sforzo di inscriversi, cucendo e riparando, proprio in quel linguaggio che egli vorrebbe padroneggiare. La lettera come margine tra senso e non-senso, tra sapere e vivere, è luogo di massima intimità del soggetto ma, al contempo, il suo punto di massima estraneità a sé stesso. Il corpo è balcone, luogo del vedere $\mathrm{e}$ dell'essere visti, delimitazione in atto tra esterno e interno, proprio come la lettera.

Prendiamo ad esempio questa frase di Tutti $i$ nostri ieri: "Anna non voleva pensare ad Ippolito sulla panchina. Si strappava quella panchina dagli occhi, aveva paura che il bambino si sentisse male se lei singhiozzava» (GINZBURG 1996, 194). La ragazza si configura come contenitore e contenuto. Incinta di Giuma, vuole dimenticare la morte per suicidio di Ippolito, trovato senza vita nel parco cittadino. Corporea è l'immagine di lei che si strappa dagli occhi la panchina, pubblico letto di morte del fratello. Il suo corpo deve liberarsi di quell'istantanea perché dal suo interno sta per nascere una vita nuova, che deve essere preservata dal dolore almeno per il lasso di tempo in cui madre e figlio risultano una diade inseparabile. Si riconosce allora come il tratto più radicalmente salvifico della Ginzburg sia la messa in discussione del paradigma che separa la vita del corpo dalla vita della mente. Per lei mente e corpo sono solidali. Il corpo è necessario per ancorarsi a una realtà concreta e arginare l'“ideologia" o continua proiezione di pensiero che risulta mortifera senza un simile ancoraggio. In Lessico Famigliare, il ritratto dedicato a Cesare Pavese corrobora quest'intuizione: 
Pavese commetteva errori più gravi dei nostri. Perché i nostri errori erano generati da impulso, imprudenza, stupidità e candore; e invece gli errori di Pavese nascevano dalla prudenza, dall'astuzia, dal calcolo, dall'intelligenza. Nulla è pericoloso come questa sorta di errori. Possono essere, come furono per lui, mortali; perché dalle strade che si sbagliano per astuzia è difficile ritornare. Gli errori che si commettono per astuzia ci avviluppano strettamente: l'astuzia mette in noi radici più fermamente che l'avventatezza. La prudenza, il calcolo, l'astuzia hanno il volto della ragione: il volto, la voce amara della ragione, che argomenta con i suoi ragionamenti infallibili, ai quali non c'è nulla da rispondere, non c'è che assentire (GINZBURG 2014, 167).

\section{Anche Ippolito, in Tutti i nostri ieri,}

Aveva pensato a tutto, ed era morto pensando a tutto. [...] non aveva scelto niente, si era lasciato tutto aggrovigliare dai suoi pensieri così da morirne. Era morto strozzato dai suoi stessi pensieri. Emilio chiese se era un essere libero chi non pensava. Cenzo Rena gli disse di non chiedere cose sceme. Era libero chi accettava di vivere quel che c'era da vivere. Era libero chi faceva dei suoi pensieri salute e ricchezza, non chi ne faceva una trappola per rimanerci strozzato (GINZBURG 1996, 160).

Il corpo, è questa la sua inedita missione etica nella narrativa ginzburghiana, non solo permette di scegliere il proprio bene, e quindi di salvarsi. Ascoltare il corpo che parla permette anche di giudicare la realtà - quella che non si sceglie - in sede morale. La scelta fra il bene e il male, fra il giusto e l'ingiusto può e deve essere fatta ascoltando il corpo più che la ragione. La Ginzburg lo dice nel saggio Le piccole virtù, contenuto nell'omonima raccolta del 1962, quando spende alcune parole sull'educazione dei figli:

Per quanto riguarda l'educazione dei figli, penso che si debbano insegnar loro non le piccole virtù ma le grandi [...]. Trascuriamo di insegnare le grandi virtù e tuttavia le amiamo e vorremmo che i nostri figli le avessero: ma nutriamo la fiducia che scaturiscano spontaneamente nel loro animo [...], ritenendole di natura istintiva mentre le altre, le piccole, ci sembrano il frutto d'una riflessione e di un calcolo e perciò noi pensiamo che debbano assolutamente essere insegnate. In realtà la differenza è solo apparente. Anche le piccole virtù provengono dal profondo del nostro istinto, da un istinto di difesa: ma in esse la ragione parla, sentenzia, disserta, brillante avvocato dell'incolumità personale. Le grandi virtù sgorgano da un istinto in cui la ragione non parla, un istinto a cui mi sarebbe difficile dare un nome. E il 
meglio di noi è in quel muto istinto: e non nel nostro istinto di difesa, che argomenta, sentenzia, disserta con la voce della ragione (GINZBURG 2015b, 99-100).

Le grandi virtù, quelle che consentono di scegliere e di vivere eticamente nel mondo, non pertengono al campo del Simbolico, all' "ideologia” ovvero alla struttura del linguaggio comune, arbitro delle piccole virtù, ma rientrano in pieno in lalangue. Le grandi virtù coincidono con un istinto innominabile che esce dal di dentro, dal corpo. In esso c'è il meglio di noi.

Per salvarsi dal dominio della parola-pensiero è fondamentale affidarsi alla parola-corpo, che s’incarna nella realtà concreta dell'uomo sotto forma di memoria. Nella narrativa ginzburghiana il ricordo ha una doppia funzione: consola dello squallore del presente e funge da schermo per la lettura dell'avvenire. Se gli inneschi della memoria nel periodo dei 'romanzi romani' sono spesso degli oggetti concreti, che permangono anche se deteriorati, in Lessico famigliare tali inneschi coincidono con dei lemmi o dei frammenti fraseologici. Gli oggetti sono masserizie che galleggiano su un fiume in piena oppure surrogati, sostituti inadeguati di qualcosa che non c'è più o non c'è mai stato. E ognuno vi si aggrappa come all'unico salvagente capace di resistere alla mareggiata. Le persone non salvano; scappano, feriscono, crollano con i loro destini.

E la parola salva? La scrittrice, nel Lessico, attribuisce alla parola ricordata e ripetuta il più grande potere: quello di legare indissolubilmente i destini. Nel romanzo viene celebrato il raccontare, la lezione del ricordare tramite il privilegio accordato a ciò che è caduco, labile e singolare; a quelle schegge che sono solo i barlumi di quello che abbiamo vissuto. La Ginzburg attinge a ricordi già elaborati mentalmente come frammenti narrativi e operatori mnesici: «quelle frasi sono il nostro latino, il vocabolario dei giorni andati, sono come i geroglifici degli egiziani o degli assiro-babilonesi, la testimonianza d'un nucleo vitale che ha cessato di esistere ma che sopravvive nei testi, salvati dalla furia delle acque e dalla corrosione del tempo» (GINZBURG 2014, 20). 


\section{Conclusioni}

Secondo Maddalena Bergamin, firmare un romanzo equivale a portare a termine un'analisi, nel senso che, grazie alla parola detta e vissuta nel corpo, il soggetto perviene a identificarsi con il proprio sintomo, di cui pure non sa tutto. Credere che il proprio sintomo possa dire qualcosa è ciò che muove l'autore a scrivere e il soggetto a entrare in analisi. Passando per l'incredulità, ci si confronta con l'impossibilità del riconoscimento, ovvero con quella parte di reale inattingibile simbolicamente e che, nondimeno, porta i suoi effetti. Si tratta della parte che sfugge al senso, al godimento della catena significante e che costituisce il dire dell'analisi e della letteratura. Infine, una scelta si impone al soggetto.

Egli potrà propendere per una sorta di ritorno di fede, seguendo il senso del desiderio e la seduzione delle parole che potrebbero colonizzare il buco lasciato dal Reale del non senso, oppure potrà scegliere di firmare il Reale silenzioso che tappa e contraddice la verità soggettiva. Avremo allora, da un lato, una cosiddetta religione del buco, dall'altro lato quella che possiamo chiamare l'ateismo del reale (BERGAMIN 2017, 125).

Quando Natalia Ginzburg nella prefazione ai Cinque romanzi brevi e altri racconti afferma che l'enjeux della sua attività consiste nel riuscire a scrivere malgrado l'infelicità causata dal rapporto con il buco del reale opta per la seconda possibilità menzionata da Bergamin. Ogni parola vergata dalla penna della scrittrice è una presa d'atto dell'ateismo del reale e della sua intangibilità, una presa d'atto dell'assunzione del fuori senso che abita il dire del soggetto. Identificarsi col sintomo per quello che di esso non si può sapere equivale, sul piano poetico e letterario, a diffidare della verità del proprio dire e ad «accettare l'opacità dell'enunciazione che soggiace agli enunciati che ne derivano» (BERGAMIN 2017, 125).

Abbiamo già potuto constatare come la scissione linguistica fra Simbolico e Reale riconosciuta da Lacan sia operativa nella narrativa ginzburghiana sia a livello tecnico che a livello esistenziale. Da un punto di vista meramente tecnico, questa frattura si manifesta tramite lo strumento della frammentazione narrativa e di tutte le diverse prese di parola sperimentate dalla scrittrice fra 
1942 e 1984. Il dramma dell'io e dell'egli, lacerante nei romanzi torinesi, raggiunge il suo culmine in Lessico famigliare traducendosi nella discrasia parassitaria fra Natalia narratrice e Natalia personaggio. Progressivamente si riassorbe nei romanzi romani dove lo stesso registro di voce viene spalmato su tutti i personaggi: frammentare il loro punto di vista significa per l'autrice spezzettare il proprio, tramite un'angolazione e uno scivolamento continuo sulla catena dei significanti. Da un punto di vista esistenziale invece, il soggetto scisso parla al lettore attraverso le voci di Delia, Anna e Cenzo Rena, tutti intrappolati, con modalità a loro peculiari, nella cosiddetta fase dello specchio. La ricerca vana dell'integrità nell'Altro li lascerà delusi perché, come hanno capito anche Elsa e Tommasino, amare l'altro da sé significa amare la sua mancanza, il vuoto che lo scava da dentro. Ogni essere umano ha il suo buco e pretendere che l'Altro arrivi a riempire il proprio significa vanificare la possibilità di un autentico incontro. In sostanza, Natalia Ginzburg con la sua letteratura insegna che esistono tre modi per far fronte al 'buco del Reale'. In primo luogo, riconoscere al dialogo, alla conversazione e alla sottoconversazione il primato dell'incontro con l'Altro. Si tratta di un incontro mai perfetto e sempre manchevole, in cui l'inadeguatezza della parola viene bilanciata dall'ancoraggio corporeo al reale. In secondo luogo, mantenere un equilibrio, una solidarietà fra "ideologia” e fisiologia, fra mente e corpo, per far fronte alla scissione interna al soggetto e al suo problematico rapporto col mondo. Infine, celebrare nel quotidiano il perpetuarsi del ricordo, illuminando quelle schegge di storia che sono solo i barlumi delle cose vissute e che pure le contengono tutte in un canto misterioso, discreto. 
M. FLORIANI, Natalia Ginzburg. «Scrivere con il corpo» sul margine del «buco del Reale», in «altrelettere», (2021), pp. 1-23, DOI: 10.5903/al_uzh-57.

Note

${ }^{1}$ Con il termine "ideologia" intendiamo delineare il campo del linguaggio comune, ordinario e la sua struttura formale soggetta alle pressioni di un determinato potere linguistico, sociale, culturale. A questo linguaggio di sistema si contrappone lalangue, direttamente connessa al corpo pulsionale. Nella nostra analisi della narrativa ghinzburghiana l'oscillazione tra i due poli si traduce con i termini fisiologia e "ideologia": il primo, già utilizzato da Cesare Garboli (GARBOLI 2012, 6), modella per assonanza il secondo.

${ }^{2}$ La nozione di lalangue è compiutamente teorizzata in J. Lacan, Le séminaire. Livre XX. Encore. 1972-1973. Il neologismo nasce dall'unione del verbo latino lallare e dal nome francese langue: la lallazione corrisponde da un lato alla melodia cantata per far addormentare un bambino; dall'altro alle prime sillabe da lui/lei pronunciate nel momento che precede il controllo del linguaggio.

${ }^{3}$ Così Cesare Garboli definisce i romanzi scritti da Natalia Ginzburg nel decennio 1970/1980.

4 Così Cesare Garboli definisce i romanzi del periodo torinese scritti da Natalia Ginzburg nel decennio 1950/1960. 


\section{Bibliografia}

BARTHES 1984:

Roland BARTHES, De l'oeuvre au texte (1971), Le bruissement de la langue. Essais critiques IV, Paris, Seuil, 1984.

BERGAMIN 2017:

Maddalena BERGAMIN, Il corpo nella lettera. Le tracce del femminile nella poesia italiana contemporanea. Le voci di Anedda, Cavalli e Gualtieri, tesi di dottorato, Université Paris-Sorbonne, Alma Mater Studiorum Università di Bologna, a.a 20162017, relatori D. Luglio, M.A Bazzocchi.

\section{CALVINO 2015:}

Italo CALVINO, «Introduzione», in N. Ginzburg, Le voci della sera (1961), Torino, Einaudi, 2015.

\section{GARBOLI 1993:}

Cesare GARBOLI, «Introduzione», in N. Ginzburg, Cinque romanzi brevi e altri racconti (1964), Torino, Einaudi, 1993.

\section{GARBOLI 1999:}

Cesare GARBOLI, «Appendice», in Natalia Ginzburg, Lessico Famigliare (1963), Torino, Einaudi, 2014.

\section{GARBOLI 2012:}

Cesare GARBOLI, «Introduzione», in N. Ginzburg, La strada che va in città (1945), Torino, Einaudi, 2012.

GINZBURG 1996:

Natalia GINZBURG, Tutti i nostri ieri (1952), Torino, Einaudi, 1996.

\section{GINZBURG 2005:}

Natalia GINZBuRg, Cinque romanzi brevi e altri racconti (1964), Torino, Einaudi, 2005 .

GINZBURG 2014:

Natalia GINZBURG, Lessico famigliare (1963), Torino, Einaudi, 2014.

GINZBURG 2015a:

Natalia GINZBURG, Le voci della sera (1961), Torino, Einaudi, 2015. 
GINZBURG 2015b:

Natalia GINZBURG, Le piccole virtù (1962), Torino Einaudi, 2015.

GINZBURG 2019:

Natalia GINZBURG, La città e la casa (1984), Torino, Einaudi, 2019.

LACAN 1973:

Jacques LACAN, Livre XI. Les quatre concepts fondamentaux de la psychanalyse. 1964, Paris, Seuil, 1973.

\section{LACAN 1975:}

Jacques LACAN, Livre XX. Encore. 1972-1973, Paris, Seuil, 1975.

SCARPA 2019:

Domenico SCARPA, «Città, strada, famiglia, casa», in Natalia Ginzburg, La città e la casa (1984), Torino, Einaudi 2019.

SEGRE 2014:

Cesare SEGRE, «Introduzione», in Natalia Ginzburg, Lessico Famigliare (1963), Torino, Einaudi, 2014.

\section{Siti Web}

\section{FERRERO 2016:}

<www.lastampa.it/cultura/2016/o7/14/news/natalia-ginzburg-la-primaminimalista-1.34835187? refresh ce> sito web ufficiale La Stampa (14.06.2016). 